\title{
La présentation des objets africains dans DOCUMENTS (1929/1930), magazine illustré
}

The presentation of African objects in DOCUMENTS (1929-30), an illustrated magazine

\section{Coline Bidault}

\section{(2) OpenEdition} Journals

Édition électronique

URL : http://journals.openedition.org/cel/500

DOI : $10.4000 /$ cel. 500

ISSN : 2262-208X

Éditeur

École du Louvre

\section{Édition imprimée}

Date de publication : 1 octobre 2013

Référence électronique

Coline Bidault, «La présentation des objets africains dans DOCUMENTS (1929/1930), magazine illustré », Les Cahiers de l'École du Louvre [En ligne], 3 | 2013, mis en ligne le 01 octobre 2013, consulté le 30 avril 2019. URL : http://journals.openedition.org/cel/500; DOI : 10.4000/cel.500

\section{c) ()ㅇㅇ}

Les Cahiers de l'École du Louvre sont mis à disposition selon les termes de la licence Creative Commons Attribution - Pas d'Utilisation Commerciale - Pas de Modification 4.0 International. 


\section{Cahiers de l'École du Louvre recherches en histoire de l'art, histoire des civilisations archéologie, anthropologie et muséologie \\ Numéro 3. Octobre 2013}

\section{La présentation des objets africains dans DOCUMENTS (1929/1930), magazine illustré}

\section{Coline Bidault}

Article disponible en ligne à l'adresse :

http://www.ecoledulouvre/revue/numero3octobre2013/Bidault.pdf

Pour citer cet article :

Coline Bidault, «La présentation des objets africains dans DOCUMENTS (1929/1930), magazine illustré », Cahiers de l'École du Louvre, Recherches en histoire de l'art, histoire des civilisations, archéologie, anthropologie et muséologie [en ligne] $n^{\circ} 3$, octobre 2013, p. 5 à 13.

Cet article est mis à disposition selon les termes de la Licence Creative Commons Attribution - Pas d'utilisation commerciale - Pas de modification 3.0 non transposé. 


\section{Cahiers de l'École du Louvre recherches en histoire de l'art, histoire des civilisations archéologie, anthropologie et muséologie \\ Numéro 3. Octobre 2013}

\section{Sommaire}

\section{Éditorial}

Équipe de recherche

\section{Dossier: les revues}

Le « GR05 ». Approche de quelques revues du XX $\mathrm{XX}^{\mathrm{e}}$ siècle Introduction d'Hélène Klein

La présentation des objets africains dans DOCUMENTS (1929-1930), magazine illustré

Coline Bidault

Le conflit renaissant de la figure et de l'abstraction dans Labyrinthe, journal mensuel des Lettres et des Arts (octobre 1944-décembre 1946)

Blandine Delhaye

BROOM: An International Magazine of the Arts (1921-1924):

une revue d'avant-garde américaine

Ambre Gauthier

L'image de la guerre dans L'Élan (1915-1916), un refoulement apparent Hadrien Viraben

\section{Articles}

" John Constable et le statut de l'esquisse »

Conférence du 9 avril 2013 dans le cadre du séminaire doctoral

John Murdoch

«Adieu veau, vache, cochon, couvée... » La boucherie à l'Ancien Empire : croisement des données iconographiques, textuelles et archéologiques

Fanny Hamonic

L’entreprise patrimoniale de Louis-Joseph GUYOT (1836-1924)

à Dourdan, entre érudition et médiation

Léda Martines

p. $63-72$

\section{Actualité de la recherche}

Reconnaissance et médiation d'un patrimoine : vecteurs d'une identité territoriale? Journées d'étude de troisième cycle, 14 et 15 juin 2012

Noémie Couillard, Nicolas Navarro, Maylis Nouvellon p. $73-81$

Les « Ymagiers » à l'École du Louvre

Cycle de conférences organisées par l'IRHT 


\section{La présentation des objets africains dans DOCUMENTS (1929/1930), magazine illustré}

\section{Coline Bidault}

Documents, magazine constitué de seulement quinze numéros parus en 1929 et $1930^{1}$, marque une rupture par rapport aux publications périodiques préexistantes. Ni revue d'art, ni revue scientifique, Documents est défini par ses fondateurs comme «l'Encyclopédie du XX $\mathrm{XX}^{\mathrm{e}}$ siècle » dont l'objectif principal est de « donner aux faits une portée nouvelle ${ }^{2} »$. Même si dans les thèmes abordés la dimension artistique prédomine, les faits en question dépassent largement le domaine de l'art. Documents, comme le montre la deuxième partie de son titre (fig. 1), se veut une publication transversale qui abolit les frontières entre les différents champs disciplinaires. Doctrines, variétés, archéologie, beaux-arts, ethnographie : tous les sujets y sont abordés et juxtaposés sans qu'aucune hiérarchie ne s’impose entre eux. Le thème de l'Afrique y est donc traité, comme tous les autres sujets, de manière transversale et pluridisciplinaire. Cette méthode, propre à ce qui se présente comme un « magazine illustré », vient interroger la manière de montrer les objets africains à une période où leur statut est plus que jamais sujet à controverses.

\section{DOCUMENTS}

\section{ARCHÉOLOGIE \\ BEA UX-ARTS \\ ETHNOGRAPHIE VARIÉTÉS}

Magazine illustré

paraissant dix fois par an

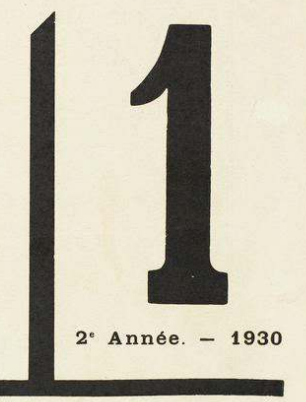

Georges BATAILLE. Le bas matérialisme et la gnose. - Marcel GRIAULE. Légende illustrée de la reine de Saba. - Jacques BARON. Jacques Lipchitz. - Jiujiro NAKAYA. Figurines néolithiques du Japon. - Robert DESNOS Pygmalion et le Sphinx.

Chronique par Georges Bataille, Arnaud Dandieu, Robert Desnos, Carl Einstein, Marcel Griaule, Michel Leiris, Georges Henri Rivière.

Photographies de Jacques-André Boiffard.

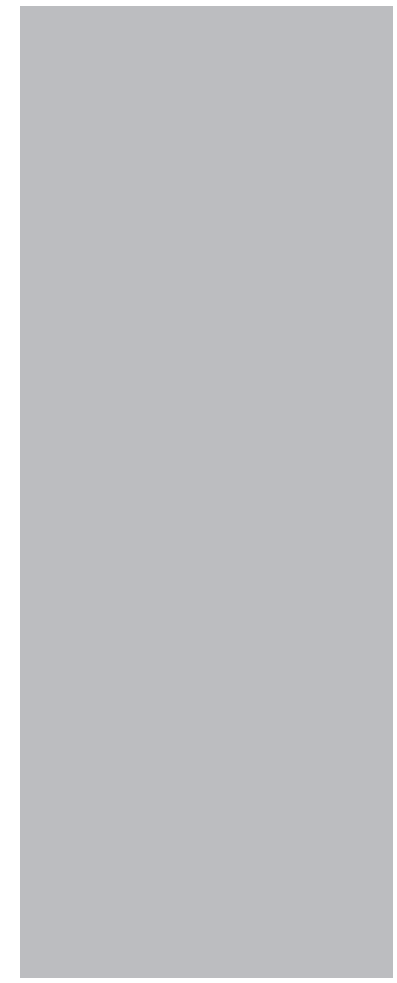

1 Documents : doctrines, archéologie, beaux-arts, ethnographie ( $\left.n^{\circ s} 1-3,1929\right)$ / archéologie, beaux-arts, ethnographie, variétés (nos 4-7, 1929 / nos 1-8, 1930), Paris, Jean-Michel Place, 1991, 396 p. (tome I) et 609 p. (tome II). Reprod. facsimilé de l'édition de Paris, [s.n.], 1929-1930. (Version numérisée disponible sur gallica.bnf.fr). http://gallica.bnf.fr/ark:/12148/cb34421975n/ date. $r=$ documents +ethnographie.langFR

2 Anonyme, sans titre, [brochure], dans Louis Yvert, Bibliographie des écrits de Michel Leiris (1924 à 1995), Paris, Jean-Michel Place, 1996, pp. 352-353. Il s'agit d'un texte publicitaire diffusé lors du lancement du magazine qui est initialement paru sous la forme d'une brochure de 8 pages au format de Documents et illustrée de cinq photographies. Il est possible d'en attribuer une partie à Georges Bataille. 


\section{Les objets africains en France à la fin des années 1920 : des œuvres d'art dans un musée d'ethnographie}

Longtemps considérés comme des témoins ethnographiques dont seule la valeur documentaire importait, les objets africains commencent à être perçus différemment au début du XX $X^{\mathrm{e}}$ siècle. À partir des années 1905-1906, ils sont prisés en raison de leurs qualités plastiques par des artistes puis des marchands d'art tels qu'Émile Heymann, Joseph Brummer ou encore Paul Guillaume, ces derniers leur conférant dès lors le statut d'objets d'art. Les "objets nègres ", tels qu'ils sont alors nommés, font des apparitions de plus en plus fréquentes au sein d'expositions, d'abord présentés en tant que source d'inspiration des œuvres modernes, puis pour leur valeur artistique intrinsèque. Cet engouement, d'abord limité à un cercle assez restreint, se développe dans les années 1920 jusqu'à devenir ce que certains qualifieront de «vogue de l'art nègre ». Pourtant, les limites d'une conception uniquement artistique des sculptures africaines sont rapidement mises en avant, notamment par des auteurs allemands tels que Carl Einstein. Ce dernier tend à montrer que même si ces objets possèdent des qualités plastiques indéniables, celles-ci sont indissociables d'un contexte de production spécifique dont la compréhension dépend d'études ethnographiques poussées ${ }^{3}$. En France, cette question sera mise en avant à l'occasion de la réorganisation du musée d'ethnographie du Trocadéro à la fin des années 1920. Cette opération, entreprise par Paul Rivet, secondé par Georges Henri Rivière, se déroule durant les deux années de publication de Documents. Elle s'effectue parallèlement au renouveau de la discipline ethnographique en France, notamment à la suite de la création de l'Institut d'Ethnologie de l'université de Paris en 1925.

\section{Renouveler la manière de présenter les objets extra-européens : du musée du Trocadéro à Documents}

Documents, et en particulier son contenu relatif à l'ethnographie et aux objets extra-européens, est en effet indissociable de la réorganisation du musée du Trocadéro, alors en pleine "déchéance » selon les termes de Georges Henri Rivière $^{4}$. Le musée n'ayant à cette période aucun organe de publication, Documents en fera office. Ainsi, le premier article concernant l'ethnographie est celui de Georges Henri Rivière à propos du musée du Trocadéro ${ }^{5}$, article qui paraitra dans la première livraison de Documents. C'est là qu'il expose sa vision du nouveau musée et les buts qu'il lui assigne ${ }^{6}$. Mais Documents se trouvera aussi être le lieu d'expression privilégié de ce qui se passe dans le monde de l'ethnologie française à la fin des années 1920 : c'est là que Paul Rivet définit ce que doit être l'étude des civilisations matérielles', mais là aussi que Marcel Griaule rendra compte de sa première mission ethnographique ${ }^{8}$ et c'est encore dans Documents que se prépare la future mission Dakar-Djibouti à laquelle participeront

\footnotetext{
${ }^{3}$ Carl Einstein écrit un premier ouvrage sur I'art africain en 1915, Leipzig, Negerplastik, Verlag der Weissen Bücher, 1915, dans lequel il s'attache à étudier les qualités formelles de l'art africain puis un second en 1921, Afrikanische plastik, Berlin, E. Wasmuth, 1921, où il s'intéresse davantage à comprendre et interpréter l'art africain en insistant sur la fonction et sur la signification des objets dans la société dont ils sont issus.

${ }^{4}$ Georges Henri Rivière, « Chronique. Le musée d'ethnographie du Trocadéro », Documents, vol. 1,1929 , p. 54.

5 Id., Ibid., pp. 54-58.

6 Un autre article à propos de la réorganisation du musée, rédigé par Georges Henri Rivière et Paul Rivet paraît en 1931 : « La Réorganisation du musée du Trocadéro », Bulletin du musée d'ethnographie du Trocadéro, vol.1, janvier 1931, p. 3. C'est aussi en 1931 qu'ils rédigent une note définissant les rôles du musée d'ethnographie c'est-à-dire un rôle scientifique, un rôle éducatif et populaire, un rôle artistique et un rôle national. Il s'agit d'une note manuscrite reproduite dans la préface de Jean Jamin à la réédition du Bulletin du musée d'ethnographie du Trocadéro : « Tout était fétiche, tout devint totem », Bulletin du musée d'ethnographie du Trocadéro, Paris, Jean-Michel Place, 1988, [1931-1935] n 1-8, p. XVII.

7 P. Rivet, « L'Étude des civilisations matérielles : ethnographie, archéologie, préhistoire », Documents, vol. 3, 1929, pp. 130-134.

8 Marcel Griaule, «Totémisme abyssin », Documents, vol. 6, 1929, pp. 316-319 et « Légende illustrée de la reine de Saba », Documents, vol. 1, 1930, pp. 9-16. A son arrivée au sein de la rédaction de Documents, Marcel Griaule revenait de la première « mission ethnographique et linguistique » qu'il a menée en Éthiopie en tant que chargé de mission à partir de septembre 1928 et pour une durée de quelques mois.
} 
plusieurs des contributeurs du magazine9. L'importance croissante du nombre d'articles concernant l'Afrique sur les deux années de publication s'amorce dans les premiers numéros à travers des textes relatifs à l'ethnographie, réflexions qui découlent directement du cadre institutionnel du musée du Trocadéro. Leurs auteurs sont tous rattachés au musée : Paul Rivet, qui en est le directeur, Georges Henri Rivière, alors sous-directeur, et André Schaeffner, responsable du département d'ethnomusicologie à partir de 1929.

Ce lien privilégié entre le cercle de l'ethnologie française et Documents amène nécessairement à faire un parallèle entre les objectifs assignés par Georges Henri Rivière au musée du Trocadéro et le contenu du magazine. Suite à l'élection de certains des objets conservés au Trocadéro au rang de chef-d'œuvre de l'art, il s'avérait pour lui nécessaire de repenser leur place au sein d'un musée d'ethnographie. Tout en s'opposant à les juger seulement sur leurs qualités plastiques, Georges Henri Rivière se propose de trouver un compromis. Il présente le musée d'ethnographie comme un tableau dont l'objectif est de « devenir un musée utile à la science et au pays, aimé des artistes et attrayant pour le public $»^{10}$. L'enjeu, bien plus que de s'occuper de l'état de conservation des collections ethnographiques françaises, est assurément de redorer le blason de l'ethnographie et de ses témoins matériels auprès du public sans pour autant perdre toute légitimité scientifique. Georges Henri Rivière précise ainsi que le nouveau musée du Trocadéro ne sera pas un musée des Beaux-arts dans lequel « les objets se répartiraient sous l'égide de la seule esthétique $»^{11}$, mais il cherche quand même à les présenter de manière à les rendre accessibles à tous et à mettre en valeur les objets les plus importants. Il souhaite ainsi que ne soient exposés dans les salles ouvertes au public que « les unica, les pièces les plus caractéristiques ${ }^{12}$ » et que les doubles et les séries soient conservés dans les magasins dont l'accès est réservé aux « travailleurs qualifiés ». La présentation des objets africains dans Documents rejoint donc les enjeux soulevés lors de la réorganisation du musée du Trocadéro puisque, dès les débuts du magazine, ses fondateurs s'attachent à trouver comment conserver une légitimité scientifique tout en évitant l'aridité des publications ethnographiques et surtout comment traiter de faits artistiques sans pour autant être une simple revue d'art.

\section{Magazine illustré / revue d'art}

Avant même sa parution, il est admis que Documents ne sera pas une revue d'art puisque ce type de publication est considéré par ses fondateurs comme un cadre bien trop restrictif au vu des vastes objectifs qui lui sont assignés. Ceux-ci sont résumés dans un texte publicitaire paru avant le lancement de Documents qui précise bien : «DOCUMENTS ne sera pas une revue d'art conventionnelle mais un magazine pratique $»^{13}$. La « revue d'art » comme le «musée des Beaux-arts » sont tous deux des modèles considérés comme dépassés dans la mesure où ils échouent à appréhender toute l'étendue des productions matérielles existantes. C'est ce mépris des institutions « conventionnelles » de l'art que l'on retrouve dans la notice de Georges Bataille sur le musée qu'il définit ironiquement comme «le miroir colossal dans lequel l'homme se contemple enfin sous toutes les faces, se trouve littéralement admirable et s'abandonne à l'extase exprimée dans toutes les revues d'art $\gg^{14}$. Ce rejet peut expliquer que Documents soit défini par ses fondateurs non pas comme une revue mais comme un «magazine illustré », mention présente sur la couverture de chaque numéro ${ }^{15}$.

\footnotetext{
9 M. Leiris et G. H. Rivière, « L'CFil de l'ethnographe (à propos de la Mission Dakar-Djibouti) », Documents, vol. 7, 1930, pp. 407-414. Organisée par I'Institut d'Ethnologie et le Muséum d'histoire naturelle de mai 1931 à février 1933, la mission Dakar-Djibouti est dirigée par Marcel Griaule et réunira deux autres collaborateurs de Documents, Michel Leiris et André Schaeffner, ainsi que le peintre Gaston-Louis Roux.

${ }^{10} \mathrm{G}$. H. Rivière, art. cité note 4, p. 58.

${ }^{11}$ Idem.

12 Id., Ibid.

13 Op. cit. note 2, p. 352.

14 Georges Bataille, «Chronique. Dictionnaire : musée », Documents, vol. 5, 1930, p. 299.

15 Documents sera qualifié à une reprise de « grande revue » dans une publicité parue dans la publication allemande Omnibus en 1931.
} 
Le choix du terme de magazine n'est sans doute pas innocent puisque ce type de publication se présente comme le seul modèle permettant de rendre compte de toute l'étendue du programme de Documents. Le magazine se différencie alors de la revue notamment par la place importante qui y est conférée à l'illustration mais aussi par son objectif de vulgarisation ${ }^{16}$. Il s'agit d'un point important pour les créateurs de Documents, qui, sans vouloir pour autant simplifier les informations, cherchent à les rendre « intelligibles » au plus grand nombre. Ils partent du principe que les publications existantes sur les sciences humaines ne remplissent pas cet objectif et restent uniquement destinées à une élite intellectuelle et déclarent quant à eux souhaiter s'éloigner de « la pédanterie prétentieuse [...] de la plupart des magazines actuels $»^{17}$. Outre les railleries proférées par Bataille à ce sujet, c'est ce qui fera dire à Carl Einstein en conclusion d'un de ses articles : «On ne vulgarise pas encore assez. Et pourtant on a des revues... $\gg^{18}$. Mais le magazine, si l'on considère son étymologie, possède surtout une dimension encyclopédique et éclectique absente des revues de cette époque et encore plus des revues d'art. Dans ce sens, le choix de cette dénomination est important puisqu'il traduit l'objectif de Documents de s'intéresser à tout, sans créer de classifications ni de hiérarchie entre les informations. Le magazine renvoie aussi à l'idée d'une accumulation d'informations et d'images, qui, plus qu'un programme intellectuel, est le fondement même de Documents. Il s'agit d'un parti pris qui influe considérablement sur la manière de montrer les diverses productions matérielles et notamment les objets africains. C'est sa qualité de magazine qui permet à Documents d'englober l'ensemble des savoirs en abordant des sujets divers sans distinction de domaines ni d'origine géographique.

Même si, à cette époque, aborder le thème des objets africains était devenu une chose courante, la manière dont le fait Documents, en mettant sur le même plan toutes les disciplines, est originale puisqu'elle permet de les appréhender dans toute leur complexité. Ils étaient en effet jusqu'alors consignés dans les pages des revues, soit aux rubriques ethnographiques soit, s'ils possédaient des qualités plastiques jugées satisfaisantes au vu des critères esthétiques occidentaux, intégrés aux rubriques d'art. Dans Les Arts à Paris ${ }^{19}$, revue fondée par Paul Guillaume, les objets africains sont reproduits parce qu'ils sont considérés comme des œuvres d'art à part entière. Dans Cahiers d'art ${ }^{20}$, bien qu'une place soit réservée aux études ethnographiques, les objets africains sont clairement répartis dans deux catégories selon des critères esthétiques : les objets considérés comme des chefs-d'œuvre sortent automatiquement du domaine de l'ethnographie, dissociation qui n'existe pas dans Documents.

\footnotetext{
${ }^{16}$ Le Trésor de la langue française informatisé définit le magazine comme une «Publication périodique généralement illustrée, traitant le plus souvent dans un but de vulgarisation de sujets divers ou d'un domaine précis » et cite l'encyclopédie française « La Civilisation écrite » datant de 1939 : « Nous avons aujourd'hui l'hebdomadaire qui garde l'aspect du journal mais qui fournit une matière plus riche et se donne un peu plus de recul pour juger les faits et les hommes. Enfin, non loin du journal et distinct de la revue, il faut placer le magazine. C'est une publication périodique, illustrée le plus souvent. Elle réserve des places variables à l'information, à l'actualité. », Encyclopédie Française, t. XVIII, La Civilisation écrite, Julien Cain (dir.), Société De Gestion De L'encyclopédie Française 1939, pp. 32-33.

17 Encart publicitaire paru dans Omnibus en 1931.

${ }^{18}$ C. Einstein, «Chronique. Exposition de collages (galerie Goemans) », Documents, vol. 4, 1930, p. 244.

${ }_{19}$ Les Arts à Paris : actualités critiques et littéraires des arts et de la curiosité, Paul Guillaume (dir.). Paris, [s. n.], 1918-1935.

${ }^{20}$ Cahiers d'art, Christian Zervos (dir.), Paris, Cahiers d'art, 1926-1960.
} 


\section{La place des objets africains dans l'iconographie de Documents}

Plus qu'un simple magazine, Documents est donc un « magazine illustré », et de cette spécificité résulte une esthétique fondée sur la juxtaposition et la confrontation des textes et des images. De par la mise en page particulière de Documents, les reproductions d'objets africains ne sont pas seulement là pour illustrer l'article auquel elles se rattachent, elles interfèrent aussi avec d'autres textes et d'autres illustrations. Contrairement à la plupart des publications de l'époque où les illustrations sont généralement regroupées, et surtout, où les illustrations traitant de l'Afrique ne se mélangent pas à celles des autres domaines, elles sont dans Documents sans cesse confrontées à des thèmes et à des types de représentations hétéroclites. Comme l'indique le titre du magazine, les objets africains, tout comme les autres objets reproduits et les différents textes publiés, sont des « documents », qui ont tous le même statut.

FIGURE 2 :
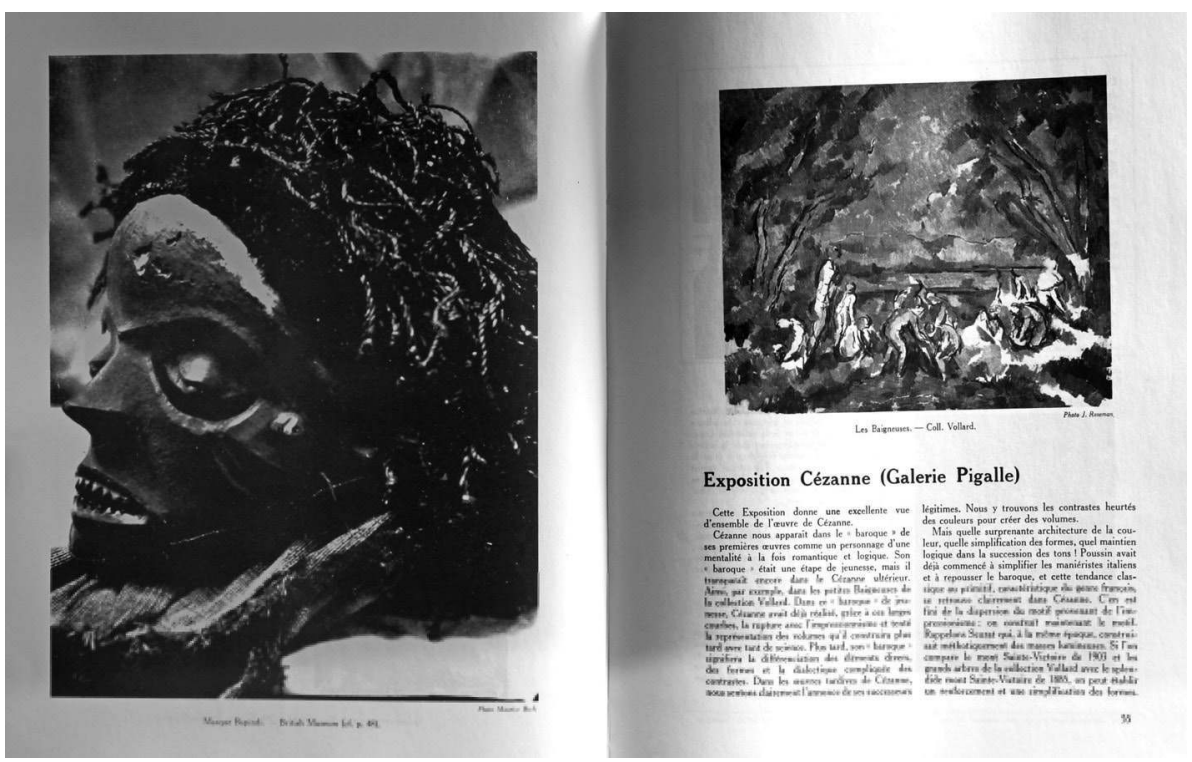

Ainsi, la photographie d'un masque bapindi, illustration d'un article de Carl Einstein à ce sujet, est placée plusieurs pages après celle du texte de l'article ${ }^{21}$. Celui-ci prend place à côté d'un paysage de Lorenzetti alors que la reproduction en pleine page du masque bapindi fait face à un article sur Cézanne (fig. 2). Passer du texte à l'image implique de traverser un article sur les mâts totémiques accompagné de trois photographies, ainsi qu'un article sur les couvertures illustrées de Fantômas dont quatre exemplaires sont reproduits. Il s'agit d'un exemple représentatif de l'enchevêtrement des sujets et des images propre à Documents qui mélange volontairement l'imagerie populaire moderne, la peinture italienne de la Renaissance et les objets extra-occidentaux. Un mélange des genres mais aussi des époques et des origines géographiques dont le masque bapindi n'est qu'un maillon.

De la même manière, les reproductions des "arbres fétiches » du Bénin, illustrations d'un autre article de Carl Einstein ${ }^{22}$, sont placées entre une page qui reproduit une partition de Georges Gershwin et une autre dévolue à des tableaux de Dalí (fig. 3). Ainsi, plus qu'un simple intérêt pour l'apparence ou la valeur de témoins de ces objets, force est de constater qu'ils prennent place dans un discours global, et ne peuvent en aucun cas être compris séparément des autres articles du magazine. En effet, les objets africains participent au vaste programme de Documents puisqu'ils s'inscrivent pleinement dans une atmosphère propre à ce magazine.

\footnotetext{
${ }^{21}$ C. Einstein, « Masques bapindi », Documents, vol. 1, 1930, pp. 48 et 54 (ill.).

22 «Chronique. Les arbres fétiches du Bénin », Documents, vol. 4, 1929, pp. 228 (ill.) et 230. Sans nom d'auteur mais attribué à Carl Einstein.
} 


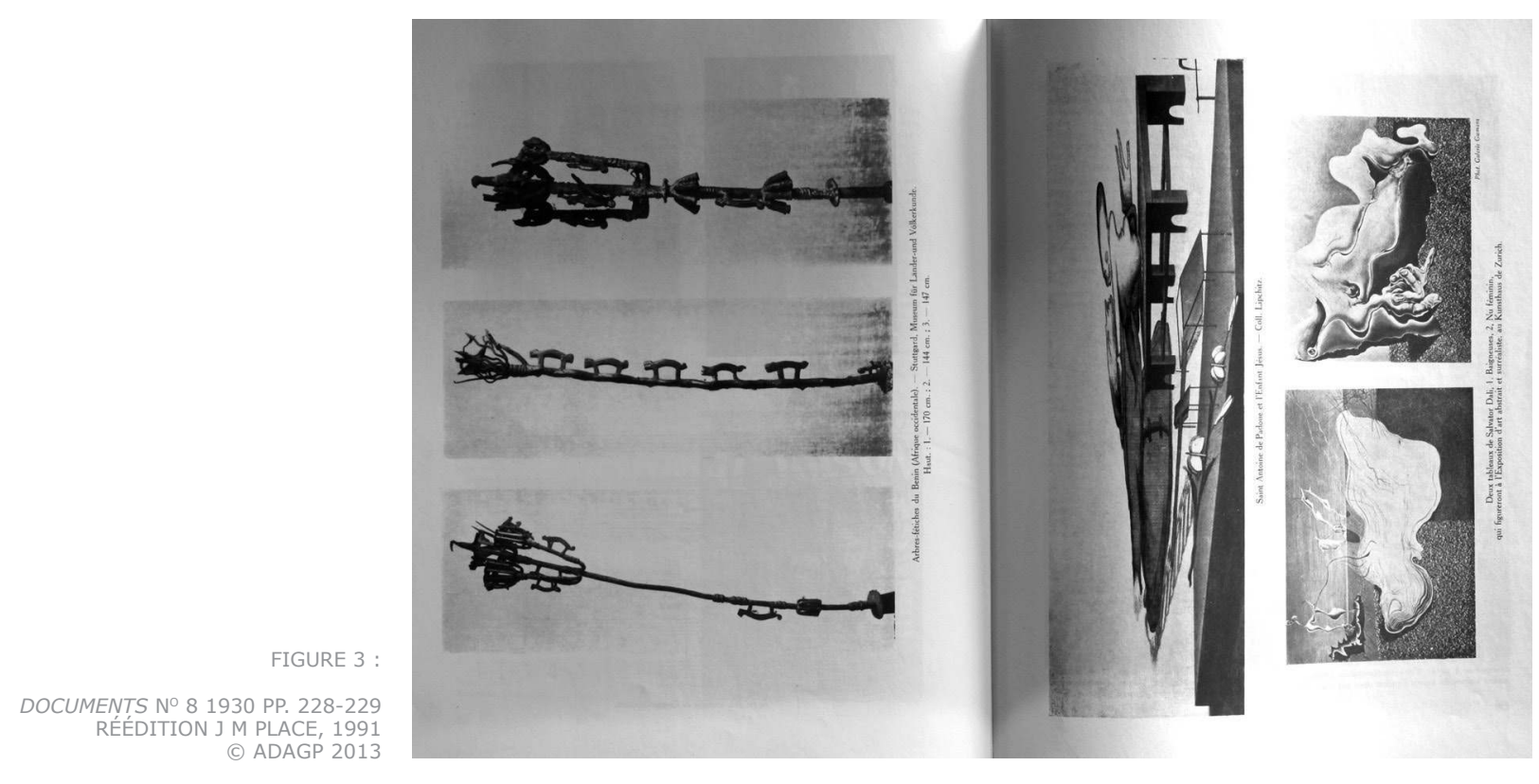

Un exemple frappant est celui de l'article d'Eckart von Sydow illustré de nombreuses reproductions de masques du Cross-River ${ }^{23}$. Considéré séparément, il s'agit d'un article sérieux rédigé par un historien de l'art allemand reconnu qui applique une analyse psychanalytique à l'étude de ces masques dont la particularité est d'avoir deux faces. Même si la valeur scientifique de l'article est incontestable, la manière dont il est intégré au reste du magazine montre que ce n'est pas seulement cet aspect qui a déterminé le choix de le publier dans Documents. Lorsqu'on le replace dans l'ensemble de la revue, la question de l'interprétation des masques-Janus dépasse largement le domaine de l'ethnographie et s'inscrit dans cette curiosité propre à Documents pour tout ce qui est considéré comme étrange, anormal et inexplicable. Cet article est ainsi placé juste avant un texte sur les monstres dans l'œuvre de Piero di Cosimo ${ }^{24}$, article illustré de plusieurs reproductions, qui selon l'auteur, peuvent être analysées comme une révolte contre la civilisation ${ }^{25}$. Ainsi, les objets africains, plus que de simples documents ethnographiques, participent de l'esthétique même du magazine au même titre que les monstres de Piero di Cosimo.

\section{Quel statut pour les objets africains dans Documents?}

L'usage particulier auquel est soumise l'iconographie dans Documents n'est pas sans conséquence sur la réception des objets africains. En jouant avec l'idée que l'on se fait de la revue d'art, mais aussi avec l'approche ethnographique, elle engendre une lecture ambiguë de des objets représentés. À de nombreuses reprises, les auteurs clament leur volonté de se détacher de tout parti pris esthétique. En témoigne la définition de l'esthète par Georges Bataille : «Étant bien entendu que personne n'adopte maintenant une telle dénomination, il faut cependant reconnaître que ce mot s'est dévalorisé de la même façon qu'artiste ou poète $»^{26}$; mais aussi le reproche que Marcel Griaule fait à ces mêmes esthètes de ne s'intéresser qu'au contenant et non pas au contenu ${ }^{27}$. Malgré la diversité des points de vue, la plupart des auteurs s'accordent sur le fait qu'il faut dépasser une approche purement esthétique des objets africains. Pourtant, de nombreuses contradictions amènent à s'interroger sur le statut réellement conféré à ces objets à travers leur reproduction dans Documents. Ainsi, bien qu'à l'échelle des articles, les reproductions d'objets apparaissent comme le support d'études ethnographiques, à l'échelle du

${ }^{23}$ Eckart von Sydow, « Les Masques-Janus du Cross-River (Cameroun) », Documents, vol. 6, 1930, pp. 321-328.

${ }^{24}$ Georgette Camille, « Piero di Cosimo », Documents, vol. 6, 1930, pp. 329-335.

${ }^{25}$ Id. Ibid., p. 335.

${ }^{26}$ G. Bataille, «Chronique. Dictionnaire : esthète », Documents, vol. 4, 1930, p. 235

27 M. Griaule, «Chronique. Dictionnaire : poterie », Documents, vol. 4, 1930, p. 236. 
magazine, c'est surtout leur impact visuel qui justifie leur reproduction. Ce qui peut au premier abord passer pour de l'«anti-formalisme $»^{28}$ doit être reconsidéré puisque dans les pages du magazine, ce qui importe n'est pas tant la fonction des objets au sein des sociétés dont ils sont issus, que leur apparence ou les représentations occidentales qui y sont associées. Dans la mise en page de Documents, la photographie de masques lors d'une danse Makishi ne fait pas qu'illustrer la description de ce rite, elle vient aussi compléter l'article suivant à propos des dessins de Cami (fig. 4) : les personnages de la danse makishi font écho à ceux des «contes à dormir debout » de $\mathrm{Cami}^{29}$.

FIGURE 4

DOCUMENTS N 81930 PP. 494-495 RÉÉDITION J.M. PLACE, 1991 (c) ADAGP 2013

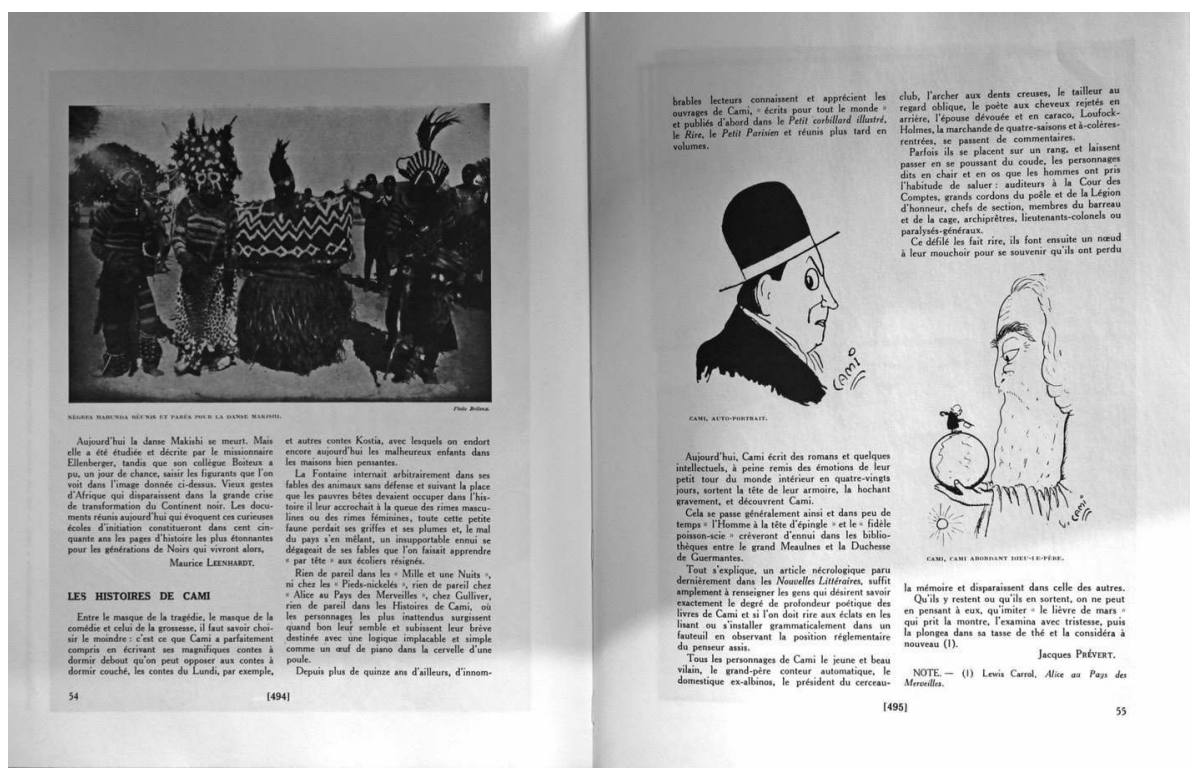

De la même manière que leur reproduction à côté d'œuvres d'art moderne permet de remettre en cause la définition des canons artistiques occidentaux, la présence de ces objets dans les pages de Documents amène le lecteur à réfléchir à la prétendue infériorité des civilisations dites alors « primitives ». Ils sont là, associés à des textes au contenu éclectique, pour comparer et relativiser : en effet, nous pouvons lire dans le texte de lancement de Documents que «les objets d'art ne sont pas des faits isolés » et qu'« aux études qui leur seront consacrées seront joints des articles sur des faits qui jouent dans la vie humaine un rôle analogue : un mythe, un système symbolique ou magique, un état psychologique particulier, un aspect du corps humain, une vie extraordinaire $»^{30}$. Le rôle des études ethnographiques auxquelles sont reliés les objets n'est donc pas tant de fournir des informations objectives sur les autres civilisations que de remettre en question la civilisation occidentale $^{31}$.

Loin du regard neutre de l'ethnographe, l'Afrique est dans Documents souvent appréhendée de manière sensible et subjective, ce qui n'est pas sans conséquences sur la façon de montrer les objets qui en sont issus. Aux yeux de la plupart des auteurs, l'Afrique est un territoire imaginaire et idéalisé et cela notamment parce que nombre d'entre eux n'y sont encore jamais allés. Ce continent fantasmé est ainsi imaginé comme un endroit où la magie règne en maître et où les hommes entretiennent encore une relation privilégiée avec le monde qui les

28 Denis Hollier, «La Valeur d'usage de l'impossible », préface du reprint de Documents publié par Jean Michel Place en 1991, p. X note 2. À propos de la phrase de Griaule - « On admirera la forme d'une anse, mais on se gardera bien d'étudier la position de l'homme qui boit » « Poterie », Documents, 1930, n 4, p. 236, Denis Hollier parle de « critique de l'esthétisme formaliste ». Selon lui, pour M. Griaule, «ce qui est essentiel dans un objet, ce n'est pas sa forme mais son usage. Et il est rarement possible de déduire l'un de l'autre. Le mode d'emploi n'est qu'exceptionnellement une conséquence de sa forme ».

29 Jacques Prévert, « Chronique. Variétés : les histoires de Cami », Documents, vol. 8, 1930, pp. 54-55 [rééd., pp. 494-495].

30 Op. cit. note 2, p. 353.

31 James Clifford, Malaise dans la culture. L'ethnographie, la littérature et l'art au XXe siècle, Paris, École nationale supérieure des beaux-arts, 1996, pp. 123-124. L'auteur parle à ce sujet de « surréalisme ethnographique » dans la mesure où selon lui l'ethnographie et le surréalisme partent à cette époque du même principe de remise en cause de la réalité. 
entoure. L'Afrique dans Documents est très souvent considérée sous l'angle du merveilleux, en tant que lieu par excellence des pratiques magiques et des rites de sorcellerie. Cette vision a des conséquences sur la manière dont sont perçues les sociétés africaines mais aussi leurs productions matérielles. Pour certains auteurs, l'Afrique possède encore un degré d'enchantement qui a disparu de la vie quotidienne de l'homme occidental ${ }^{32}$. En témoigne notamment la publication de l'article de Marcel Griaule sur le mythe totémique d'une société éthiopienne dont les membres estiment descendre d'un aloès ${ }^{33}$. Plus qu'une simple curiosité d'ethnographe, il s'agit d'un intérêt pour le rapport magique qui peut exister entre l'homme et l'animal ou la nature. Le totémisme africain rejoint l'idée du déguisement, de la métamorphose, thème abordé à de nombreuses reprises dans Documents $^{34}$. Mais le fantasme d'un humain-animal doit aussi être mis en relation avec la fascination pour l'étrange, pour les représentations inhabituelles du corps humain, ce qui explique la présence dans les pages du magazine de nombres de masques africains symboles de ce travestissement magique de l'être humain. Ces masques, appartenant pour la plupart à des collections muséales ${ }^{35}$, sont sortis de leur contexte pour être mis en rapport avec d'autres types de représentation. Dans les pages de Documents, tout comme les masques du Cross-River de l'article d'Eckart von Sydow font écho aux monstres de Piero di Cosimo ${ }^{36}$, le masque ekoi, dont il est précisé qu'il se signale " par un naturalisme presque effrayant $»^{37}$ doit être lu en parallèle avec les reproductions des mosaïques des thermes de Caracalla qui le précèdent, les deux types de représentation se rejoignant dans le rendu du corps humain (fig. 5).

列
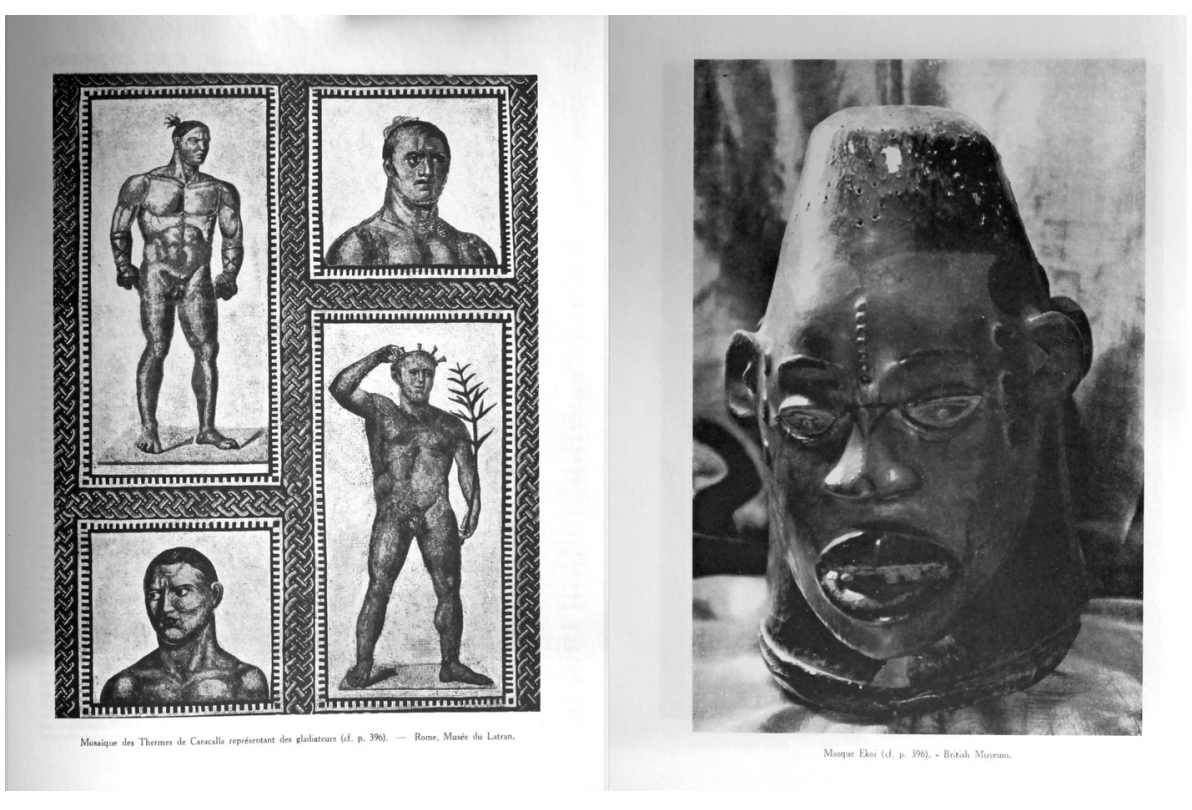

32 Ces auteurs sont pour la plupart des « dissidents » du mouvement surréaliste. La plupart des signataires du Cadavre, un pamphlet paru le 15 janvier 1930 et dirigé contre André Breton à la suite de la parution du Second manifeste du Surréalisme, ont collaboré à la revue Documents (Bataille, Ribemont-Dessaigne, Desnos, Prévert, Queneau, Vitrac, Leiris, Limbour, Boiffard). 33 M. Griaule, « Totémisme abyssin », Documents, vol. 6, 1929, pp. 316-319.

34 M. Griaule, M. Leiris, G. Bataille, « Chronique. Dictionnaire : métamorphose », Documents, vol. 6,1929 , pp. 332-334.

35 Tous les objets africains reproduits dans Documents sont issus de collections muséales sauf ceux illustrant I'article de Carl Einstein « À propos de I'exposition de la galerie Pigalle » Documents, 1930, n² 2, p. 104-112 qui appartiennent tous à des collectionneurs privés : collections de Miré, Ascher, Loeb, Ratton, Vignier, Hein.

36 Voir supra.

${ }^{37}$ C. Einstein, « Chronique. Masque de danse rituelle ekoi », Documents, vol. 7, 1929, p. 396. 
Ainsi, il n'y a pas dans Documents une seule vision de l'Afrique mais bien une multiplicité de points de vue. L'approche sensible de l'Afrique est en perpétuelle tension avec le contenu scientifique des articles « ethnographiques », et l'interprétation des reproductions est elle aussi soumise à cette hétérogénéité de contenu. En permettant l'expression de points de vue contradictoires, Documents parvient à atteindre les objectifs assignés par Georges Henri Rivière au musée du Trocadéro, voire les dépasse. En effet, la présentation des objets africains dans Documents, sans leur ôter leur statut de témoins matériels des sociétés extra-occidentales, ne leur fait pas perdre toute valeur esthétique.

Il y a dans Documents un jeu avec les normes de présentation des objets ethnographiques créant un décalage dans l'interprétation des objets africains. C'est pourquoi il n'est pas possible de parler d'une approche anti-esthétique qui se soucierait uniquement de la fonction en délaissant l'aspect formel ${ }^{38}$. Loin de se focaliser sur l'un ou l'autre de ces aspects, Documents permet plutôt d'en faire une synthèse. C'est par son absence de cohérence, souhaitée par ses fondateurs, que Documents parvient à dépasser l'opposition traditionnelle entre objets d'art et objets ethnographiques. En montrant les objets africains sous un angle original, Documents permet d'élargir la vision que l'on en avait et parvient à leur conférer cette " portée nouvelle » souhaitée à la création du magazine.

\section{Pour citer cet article}

\section{Référence électronique}

Coline Bidault, " La présentation des objets africains dans DOCUMENTS (1929/1930), magazine illustré », Cahiers de l'École du Louvre. Recherches en histoire de l'art, histoire des civilisations, archéologie, anthropologie et muséologie [en ligne] no 3 , octobre 2013.

URL : http://www.ecoledulouvre.fr/revue/numero3octobre2013/Bidault.pdf

\section{cccreative \\ (B.) $\ominus$ (\$)}

\section{L'auteur}

Après des études en Lettres modernes, en Histoire de l'art et en urbanisme à l'université de Lyon, Coline Bidault a rejoint le master 1 de l'École du Louvre en 2011 dans le cadre duquel elle a soutenu un mémoire intitulé "L'Afrique dans la revue Documents (1929/1930)» sous la direction d'Hélène Klein et de Jean-Louis Paudrat. Elle s'est ensuite intéressée, dans le cadre du master 2 professionnalisant "métiers du patrimoine », aux enjeux soulevés par l'exposition de sculptures contemporaines en plein-air.

\footnotetext{
38 Georges Didi-Huberman, La Ressemblance informe ou le gai savoir visuel selon Georges Bataille, Paris, Macula, 1995, note 1, p. 17. Pour l'auteur, il n'est pas possible de parler « d'antiformalisme » à propos de Documents. Pour lui la phrase de Griaule contre les esthètes, citée note 28, participe d'un dépassement de I'opposition classique entre la forme et la fonction, la « position » de l'homme étant relative à la forme même de l'objet.
} 